\title{
Epizootic Monitoring of Erysipeloid Foci in the Republic of Armenia, 2007-2016
}

\author{
Laura Mkrtchyan*, Armine Ghazazyan and Ruben Danielyan
}

RA NCDCP Reference Laboratory Center Branch, Laboratory of Especially Dangerous and Natural Foci Infections, Yerevan, Armenia

\section{Objective}

The goal of this study was to characterize the epidmiological, geographic, and historical characteristics of erysipeloid outbreaks in the Republic of Armenia.

\section{Introduction}

Erysipeloid is a zoonotic bacterial infection transmitted to humans from animals. Symptoms include inflamed joints and skin; there is also a generalized type of the infection in which bacteria spread through the lymphatic and blood vessels, leading to the emergence of widespread skin lesions and the formation of secondary foci of infection in internal organs. Morbidity has no age or gender specifics; there is summer and autumn seasonality. The agent of the infection - Erysipelothrix rhusiopathiae can be found in many domestic and wild animals. Wild rodents and ectoparasites play an essential role in spreading the disease and serve as a source of infection contaminating the environment.

\section{Methods}

Tests are conducted on both national and Marz levels in Reference Laboratory Center of NCDC SNCO and Marz branch laboratories of Especially Dangerous, Zoonotic and Natural Foci Infections respectively. Tests for detection of E. rhusiopathiae and confirmation of epizootics are conducted on rodents and ectoparasites collected from their hair and nests from 373 sectors of Armenia. Tests include smear microscopy and a bioassay in which cultures from a suspension of rodent organs or an emulsion of ticks and fleas are injected into white mice to assess the presence of agent in the organs or parasites.

\section{Results}

Ten years of monitoring indicates that erysipeloid epizootics have been recorded annually in Armenia with a total number of 119 cases. The most outbreaks were recorded in 2011 when 26 cases were recorded while in 2009 there were 20. The lowest number of cases recorded was five in 2008. Kotayk, Aragatsotn and Lori Marzes have the least number of cases with only 1-3 recorded epizootics, while Vayk, Gegharkunik and Shirak Marzes are considered active foci with 5-7 cases reported. Microbiological analyses indicates that $80 \%$ of cultures were isolated from field mice, $13.3 \%$ from gamasid ticks, $4.2 \%$ from fleas and $2.5 \%$ from ixodid ticks.

\section{Conclusions}

The presence of E. rhusiopathiae is stable in Armenia. It is found among rodents, where the epidemiological situation remains unfavorable. Constant regular tests/analyses are required to prevent human and animal infection. There is a need to enhance the area of test sites and apply most up-to-date methods of analysis i.e. ELISA, PCR so that the live bioassays in mice can be halted.

\section{Keywords}

erysipeloid; Armenia; epidemiology; disease monitoring

\section{*Laura Mkrtchyan}

E-mail: lara.mkrtchyan@gmail.com 